\title{
On New Polygonal Designs Constructed using Spidrons and New Tiling Patterns Generated by Them
}

\author{
T. Gangopadhyay \\ XLRI \\ C. H. Area (E), Jamshedpur, \\ India
}

\begin{abstract}
Spidrons have been constructed by repeatedly connecting the alternate vertices of a regular polygon. In the present paper new symmetric designs with inscribed regular polygons are constructed using n 6-part spidrons. Also several new tiling patterns are created using these designs
\end{abstract}

\section{General Terms}

Tiling, Algorithm, Turbo C++, Program

\section{Keywords}

Spidron, polygon, isosceles

\section{INTRODUCTION}

In an earlier paper, the present author has studied new tiling patterns with inscribed polygonal designs that are created using a fractalesque structure called spidron (Gangopadhyay([3]). In the present paper, the author studies the effect of juxtaposing two or more such spidron-based polygonal designs and using them to create new tiling patterns. An n-part spidron is a figure in plane geometry consisting entirely of an alternating sequence of two isosceles triangles, each with its own base angles[2], [5], [7]. The two triangles are juxtaposed in such a way that together they from a third bigger triangle. The sequence is then drawn at an appropriate angle on a smaller scale and the process is repeated ad infinitum. n-part spidrons have been largely studied for $\mathrm{n}=6$ and 8[4]. A regular $\mathrm{n}$-sided polygon can be split into $\mathrm{n} n$-part spidrons[6]. A 6-part spidron is displayed in Figure 1. Erdely[2], has called the same structure a semispidron.

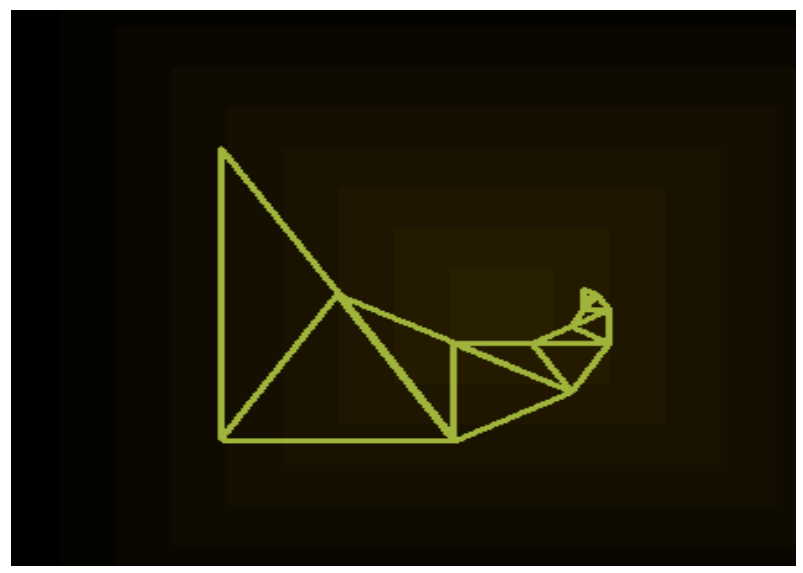

Fig 1 : A six-part spidron

In the present paper, the author presents new symmetric designs which inscribe regular polygons using $\mathrm{n}$ 6-part spidrons and explores several tiling designs with the same. Sometimes two symmetric designs are superimposed to create the basic building block for a tiling pattern. These are primarily the distinctive features of this paper.

\section{THE ALGORITHM}

For the basic building block one uses two symmetric designs both created using six 6-part spidrons. To do this one draws consecutive spidrons in a clockwise rotation for the first design and in an anti-clockwise rotation for the second design. These two designs are depicted in Figures 2 and 3 respectively. We also produce a third symmetric design by superimposing the first two. This is depicted in figure 4. The first design has been already presented in Gangopadhyay[3].

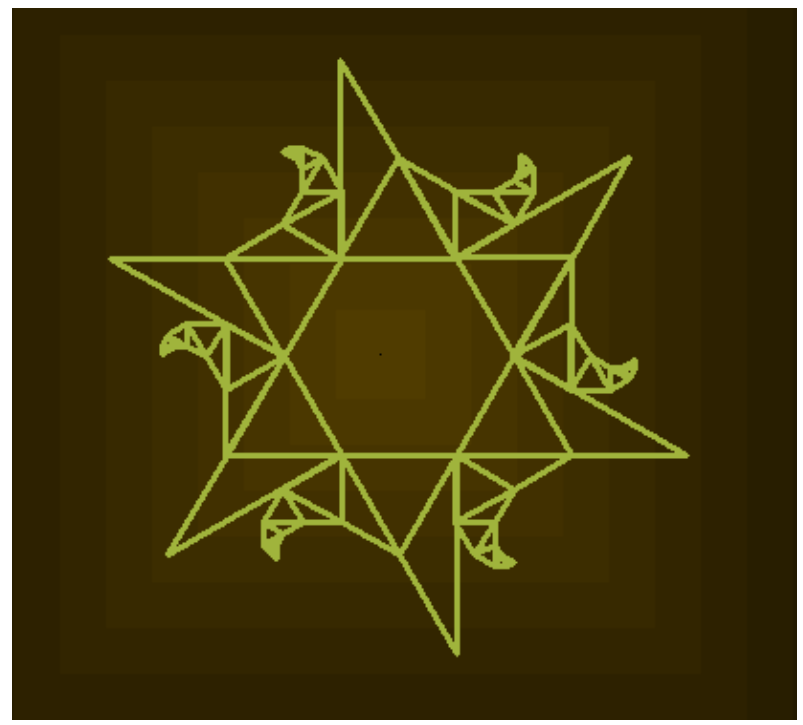

Fig 2 : The first symmetric design

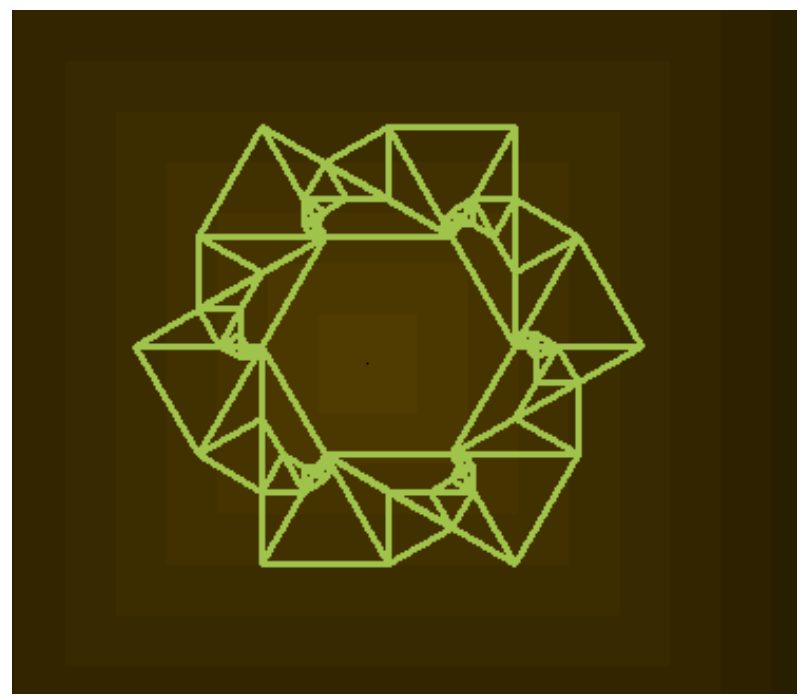

Fig 3 : The second symmetric design 


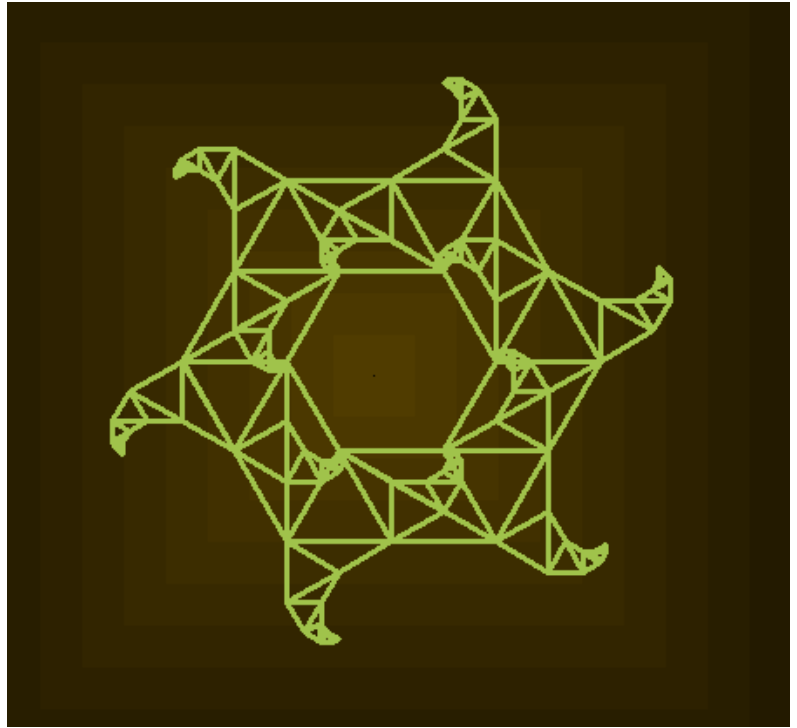

Fig 4 : The superimposed design

In the next section one submits a programming code in Visual $\mathrm{C}++$ that captures the algorithm and generates figures 2,3 and 4 .

\section{THE CODE}

The code uses two functions spid1 and spid2 which are declared first. Each function has two parameters $-s$, which gives the value of the two equal sides of the first isosceles triangle and 1 , which specifies the number of recursive iterations. Both functions draws a single n-part spidron. However, they differ in terms of the direction in which the recursive iterations occur and also in terms of the final position of the cursor, Three other functions, polyspidron1, polyspidron 2 and polyspidron 3 uses the function spid 1 and/or spid2 in a loop. Polyspidron1 uses only spid1(Figure 2), polyspidron 2 only spid2(Figure 3 ) and polyspidron 3 uses both spid 1 and spid 2 to create the superimposed polygonal design displayed in figure 4. Also both polyspidron1 and polyspidron 2 use only one right rotation, though the respective angles differ, while polyspidron 3 uses one left and one right rotation, the right rotation angle being identical to the one used by polyspidron1. The functions fd, rt, lt, pu and pd are adapted from turtle graphics[1] and have their usual connotations. The function fd draws a line of specified length, $\mathrm{rt}$ and lt respectively rotates the pen right and left by a specified angle, pu puts the pen up(no drawing) and pd puts the pen down. The various functions and the code are given below:

int $\mathrm{n}=12$;

float ang $=90, p x, p y, p s=1$;

void fd(float dist)

\{float $\mathrm{hx}=\cos ($ ang $* 3.1415926536 / 180)$;

float hy $=\sin ($ ang $* 3.1415926536 / 180)$;

float $\mathrm{nx}=\mathrm{px}-\mathrm{hx} *$ dist;

float ny=py-hy*dist;

if(ps!=1)goto label;

line(px,py,nx,ny);

label:px=nx;py=ny;
\}

float rt(float 1)

$\{$ ang+=1;

return ang; \}

float lt(float l)

$\{$ ang-=l;

return ang; $\}$

void $\mathrm{pu}()$

$\{\mathrm{ps}=0 ;\}$

void $\operatorname{pd}()$

$\{\mathrm{ps}=1 ;\}$

void spid1(float s,int 1 )

\{float a,b;

if(l==0)\{rt(90);fd(s);lt(120);fd(s);fd(s);lt(150);fd(sqrt(3.)*s); lt (150);fd(s);rt(120);fd(s);lt(60);return;\}

spid1(s,l-1);lt(120);a=px;b=py;spid1(s/sqrt(3.),l-

1);px=a;py=b;rt(30);

\}

void spid2(float s,int 1 )

\{float a,b;

if(l==0) $\left\{\mathrm{fd}\left(\operatorname{sqrt}(3 .)^{*} \mathrm{~s}\right) ; \operatorname{lt}(90) ; \mathrm{fd}(\mathrm{s}) ; \operatorname{lt}(120) ; \mathrm{fd}(\mathrm{s}) ; \mathrm{fd}(\mathrm{s}) ; \mathrm{rt}(180) ; \mathrm{f}\right.$ $\mathrm{d}(\mathrm{s}) ; \mathrm{rt}(60) ; \mathrm{fd}(\mathrm{s}) ; \mathrm{rt}(180) ; \mathrm{fd}(\mathrm{s}) ; \mathrm{rt}(120) ;$ return;\}

spid2(s,1-1);a=px;b=py;spid2(s/sqrt(3.),1-1);px=a;py=b;rt(30);

\}

void polyspidron1(float s,int l)

\{ for(int $\mathrm{i}=0 ; \mathrm{i}<\mathrm{n} ; \mathrm{i}++)\{\operatorname{spid} 1(\mathrm{~s}, 1) ; \mathrm{rt}(90-(2 * \mathrm{n}-4) * 90 / \mathrm{n}) ;\}$

\}

void polyspidron2(float s,int l)

$\{$ for(int $\mathrm{i}=0 ; \mathrm{i}<\mathrm{n} ; \mathrm{i}++)\{\operatorname{spid} 2(\mathrm{~s}, 1) ; \mathrm{rt}(-150+(2 * \mathrm{n}-4) * 90 / \mathrm{n}) ;\}$

\}

void polyspidron3(float s,int $\mathrm{l}$ )

$\{$ for(int $\mathrm{i}=0 ; \mathrm{i}<\mathrm{n} ; \mathrm{i}++)\{\operatorname{spid} 1(\mathrm{~s}, 1) ; \mathrm{bk}(\mathrm{s}) ; \operatorname{lt}(150-(2 * \mathrm{n}$ -

$8) * 90 / n) ; \operatorname{spid} 2(\mathrm{~s}, 1) ; \mathrm{rt}(90-(2 * \mathrm{n}-4) * 90 / \mathrm{n}) ;\}$

\}

void main()

\{

initwindow(1000,800);

setlinestyle(SOLID_LINE,0,THICK_WIDTH);

float $\mathrm{s}=70 ; \mathrm{px}=380, \mathrm{py}=620$;

polyspidron3(s,5);

getch();

closegraph();

\} 
The output of the sample code is illustrated in Figure 5 where $\mathrm{n}$ has been set to 12 , a superimposed design inscribing a regular dodecahedron.

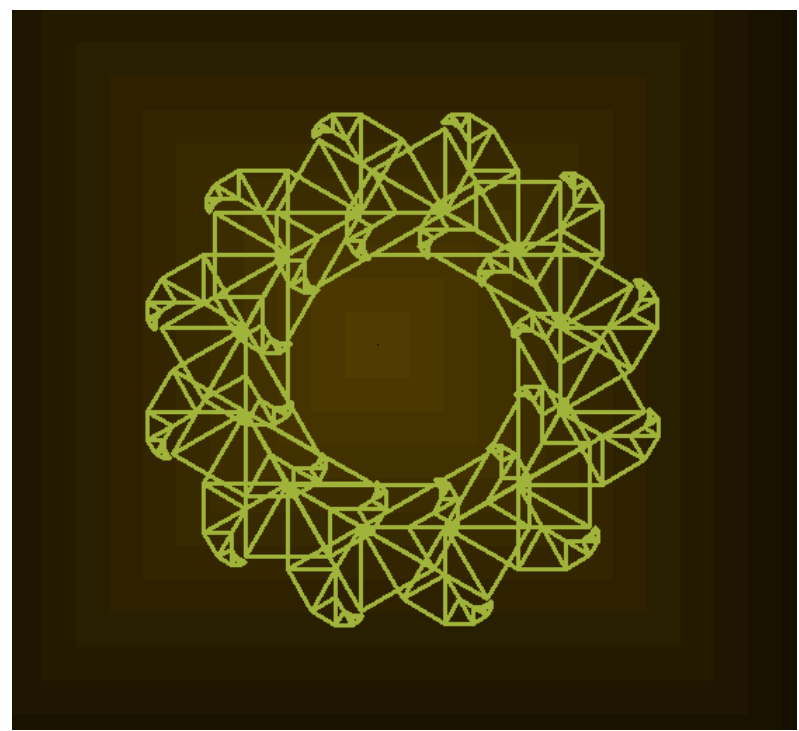

Fig 5 : Output of the sample code, $n=12$

Figures 6 and 7 depict the respective outputs for $n=4$ and $n=5$.

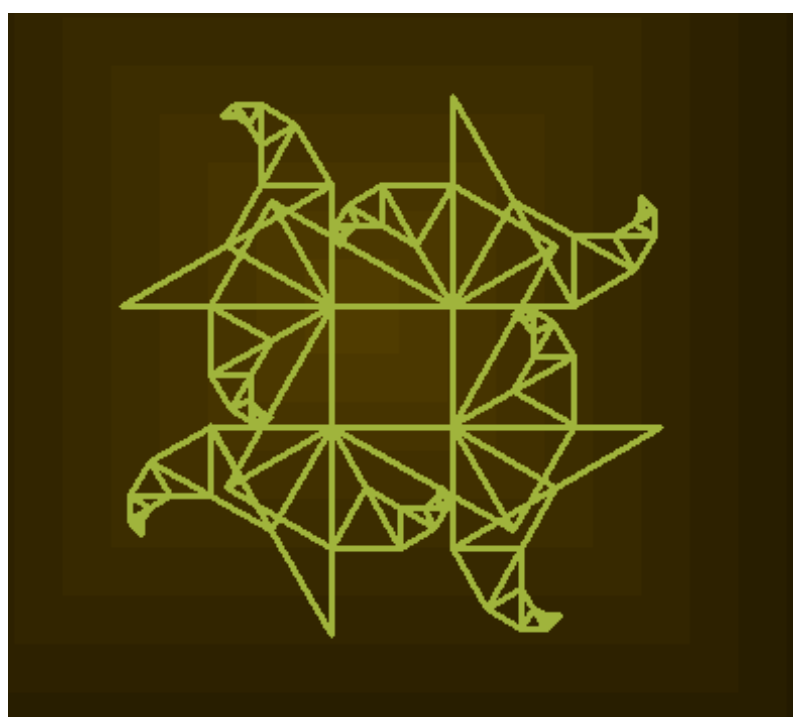

Fig. 6 : Output of the sample code for $n=4$

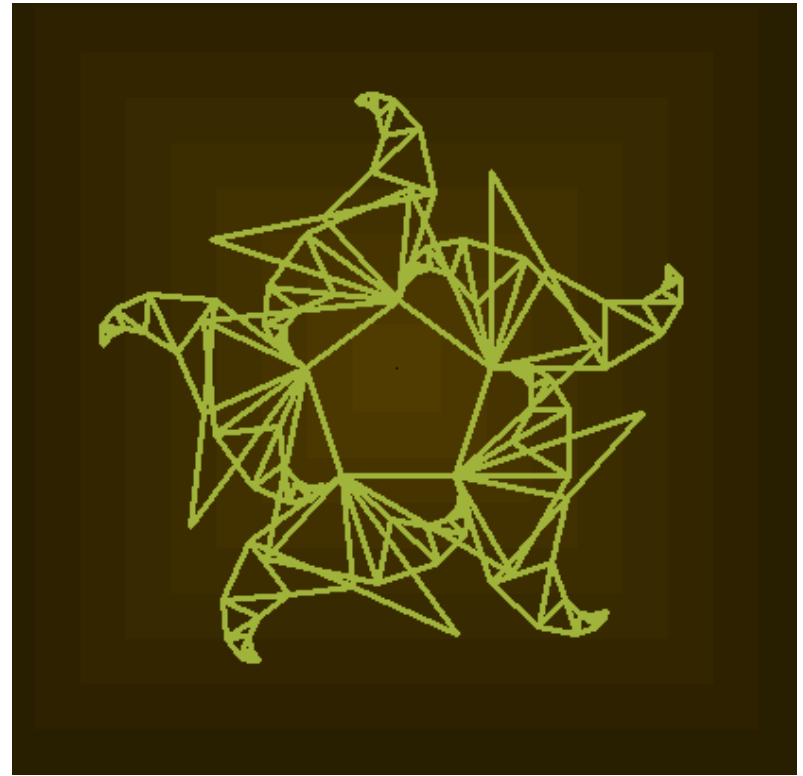

Fig. 7 : Output of the sample code for $n=5$

It can be easily observed from figures 6 and 7 that the new design will always inscribe an $\mathrm{n}$-sided regular polygon.

\section{TILING THE PLANE USING THE POLYGONAL DESIGNS}

Tiling with the first symmetric design(figure 2) has already been explored in[3]. Below we study the effect of the second symmetric design(figure 3) and that of the superimposed design(figure 4) on tiling patterns. Figures 8,9 and 10 respectively show the effect of the second symmetric design on tiling patterns for $n=6,4$ and 3, while figures 11,12 and 13 respectively show the effect of the superimposed design on tiling patterns for $n=6,4$ and 3

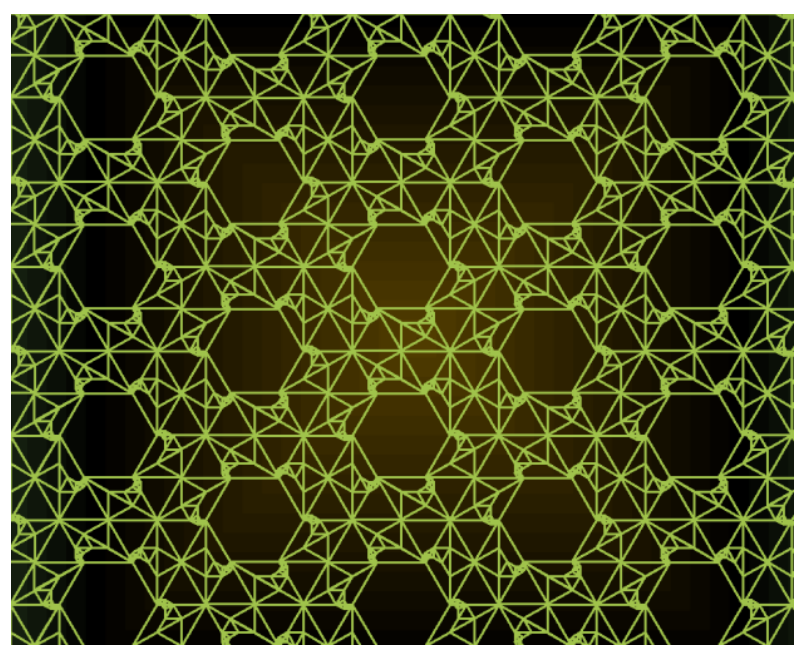

Fig. 8 : Tiling with the second symmetric design for $n=6$ 


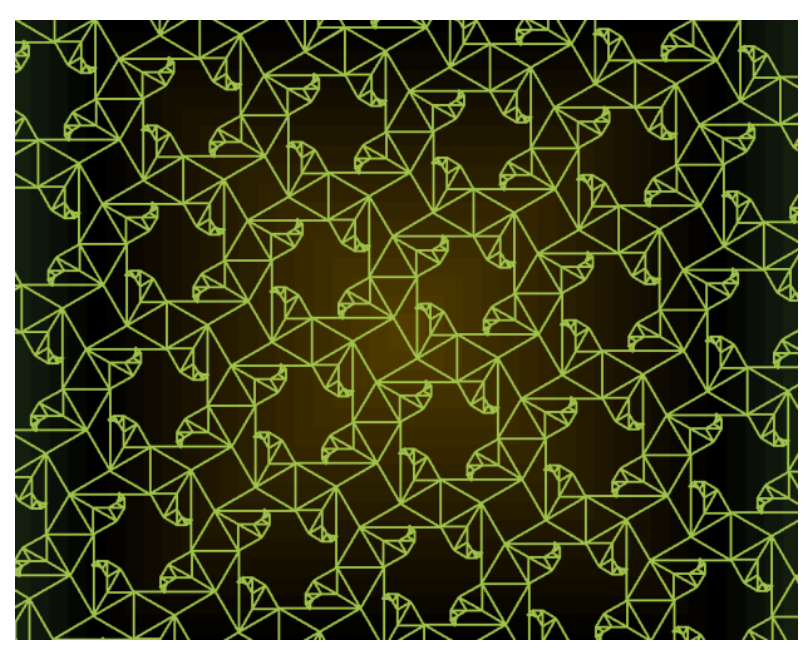

Fig. 9 : Tiling with the second symmetric design for $n=4$

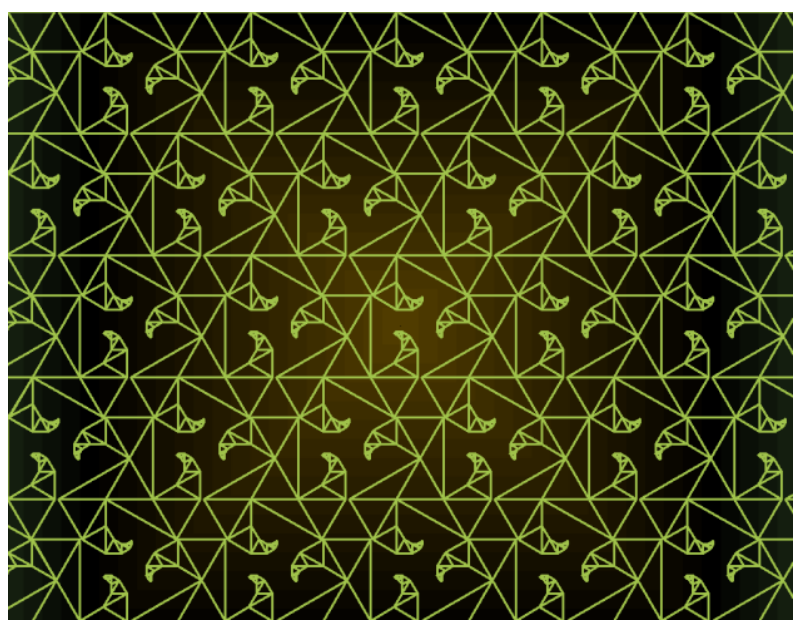

Fig.10. Tiling with the second symmetric design for $n=3$

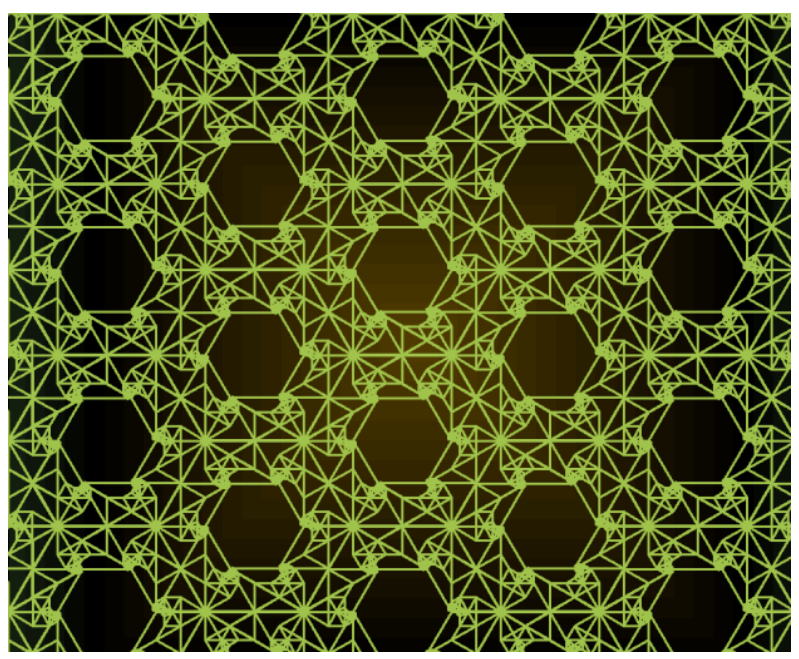

Fig. 11 : Tiling using the superimposed design for $n=6$

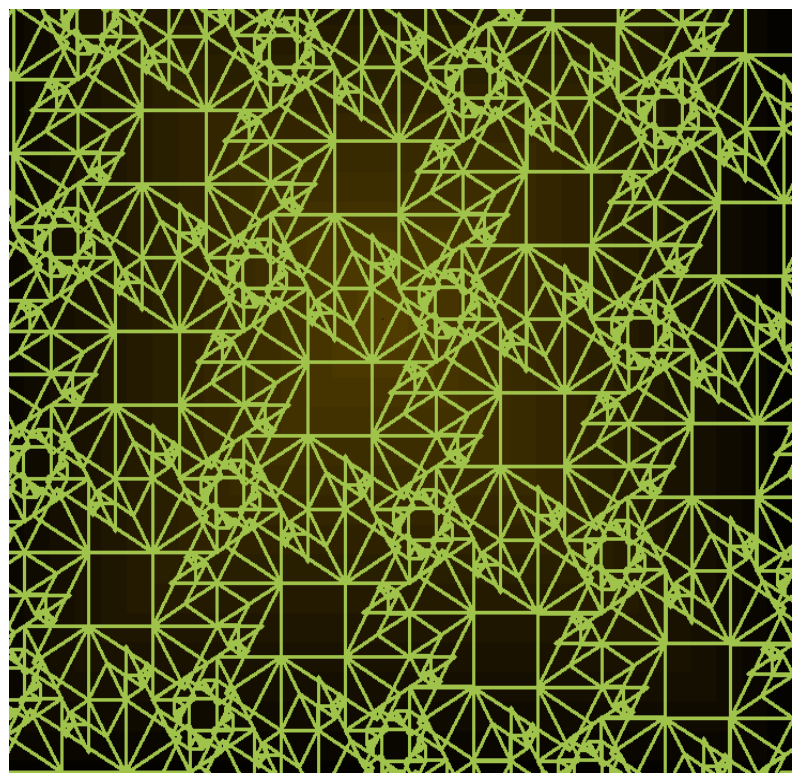

Fig. 12 : Tiling using the superimposed design for $n=4$

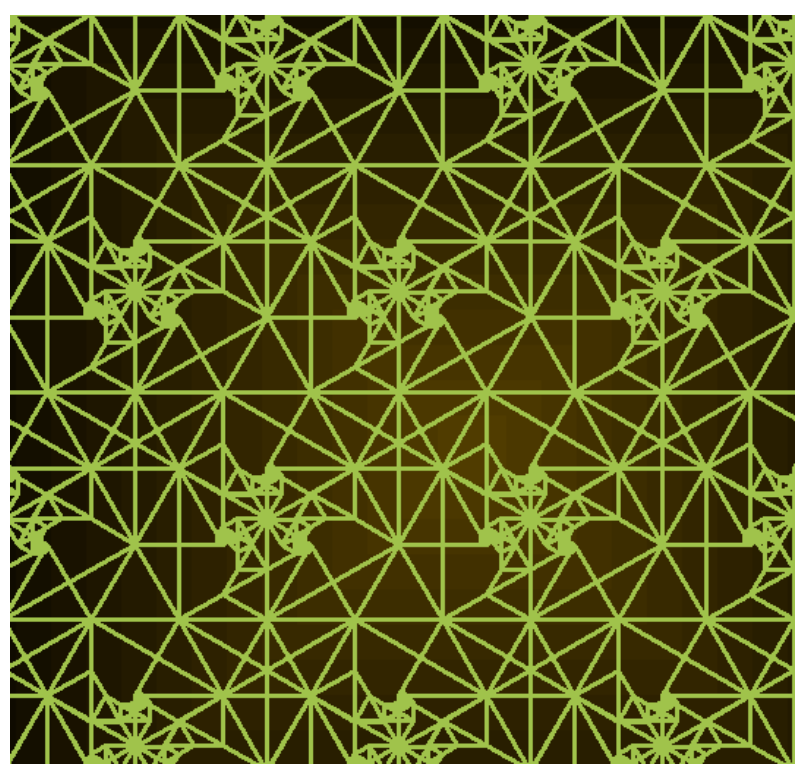

Fig. 13 : Tiling using the superimposed design for $n=3$

\section{CONCLUSION}

This paper presents new symmetric designs which inscribe regular polygons using $\mathrm{n}$ 6-part spidrons and explores several tiling designs with the same.. In subsequent studies one will further explore aperiodic tiling patterns created with polygonal designs using an odd number of spidrons. Further, the construction of new structures similar to spidrons will also be studied along with the effect of these structures on tiling patterns. These are the aspects that would be explored in future work.

\section{ACKNOWLEDGMENTS}

The author wishes to acknowledge his debt to the referee(s) for their constructive suggestions and encouragement 


\section{REFERENCES}

[1] Abelson and diSessa, Turtle Geometry, MIT Press, 1992

[2] Erdely,D.http://www.bridgesmathart.org/art exhibits/bridges2007/erdely.html.

[3] Gangopadhyay, T. On an alternate construction method for generating spidrons and new tiling patterns generated by them, International journal of Computer Applications, /volume160/number3/270542017913010
[4] Jacques, F. http://polyspidrons.over-blog.com/article4823990.html.

[5] Peterson, I. "Swirling Seas, Crystal Balls", ScienceNews.org. Archived from the original on February 28, 2007. Retrieved 2007-02-14.

[6] Stenzhorn, S. Mathematical description of Spidrons ,http://stefanstenzhorn.com/Spidrons.

[7] https://en.wikipedia.org/wiki/Spidron. 